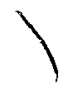

\title{
El comportamiento electoral en la megalópolis
}

\section{Boris Graizbord*}

El enfoque de la geografía electoral resulta interesante para analizar las decisiones espaciales y el comportamiento político de los individuos. En este trabajo incorporo a este enfoque el de algunos modelos analíticos de la economía y la ciencia política para interpretar los resultados electorales de 1988, para presidente de la República en la Zona Metropolitana de la Ciudad de México, y la región megalopolitana del centro del país.

El análisis empírico permite afirmar que la distribución de los votos entre partidos políticos refleja más que la estructura de clases, las diferencias espaciales en la región de estudio.

\section{Presentación}

En este trabajo me propongo evaluar los resultados electorales de 1988 , a partir del análisis de la tendencia espacial que siguió el voto presidencial y su distribución entre partidos políticos según las zonas en que he dividido el ámbito megalopolitano de la región centro (RC) del país. ${ }^{1}$ Con ello intento corroborar, para este ámbito espacial, algunas generalizaciones empíricas que aparecen en la literatura sobre el comportamiento de los electores metropolitanos.

Incorporo algunas ideas y conceptos de otras disciplinas fuera de la geografía en la formulación de mis hipótesis de trabajo. Y termino con una reflexión acerca de los condicionantes sociales que en la actualidad afectan las ideas y posiciones políticas de los ciudadanos en las grandes urbes.

\section{Un enfoque multidisciplinario}

Desde el punto de vista de la geografía política, y aceptando que la "democracia" tanto como el "bienestar social" tienen carácter

\footnotetext{
* Profesor-investigador del Centro de Estudios Demográficos y de Desarrollo Urbano de El Colegio de México.

1 Esta región incluye los estados de México, Morelos y al Distrito Federal, es decir, el envolvente geográfico y político-administrativo de la Zona Metropolitana de la Ciudad de México (ZMCM) y la llamada "megalópolis emergente" (Garza, 1987). Se subdivide en 78 distritos electorales (40 del D.F., 34 del Estado de México y 4 de Morelos) que agrupan o fragmentan (como en el caso de Nezahualcóyotl, con 10 distritos) a 16 delegaciones del D.F., 121 municipios del Estado de México y 32 de Morelos (vid infra).
} 
de bienes públicos puros, ${ }^{2}$ en una situación quid-pro-quo entre gobierno y gobernados, podría esperarse que al principio de "por cada individuo un voto" correspondiera una distribución equitativa del ingreso en todas partes (Thurow, 1971). Lo último resulta poco menos que inalcanzable dada la "natural" distribución (no homogénea) de recursos materiales y humanos en el espacio geográfico. El principio del voto, por otra parte, puede establecerse como criterio democrático a priori y elevarse a decreto constitucional, independientemente de que se cumpla en la práctica.

Desde el punto de vista de la competencia electoral, una situación como la que se observa en nuestro país, en la que el partido en el poder ha obtenido menor votación relativa en la capital, llevaría a pensar en la conveniencia política (más que en la necesidad económica) de reducir la histórica concentración de la población, las actividades económicas y los recursos en general. Una política compensatoria basada en la descentralización de oportunidades y beneficios sociales, a través de la distribución más equilibrada y justa de la inversión pública en el territorio nacional, sería una forma de lograr aquélla. Que esto se consiga y redunde además en ventajas electorales, está por verse.

Resulta difícil en la práctica convencer a los funcionarios públicos que toman decisiones y diseñan las políticas presupuestales, sobre la conveniencia de apoyar con recursos extraordinarios a aquellas áreas, entidades o ciudades donde los electores votan por los partidos de oposición, o donde el abstencionismo es muy elevado.

Como lo demuestran los recientes resultados electorales, algunos ciudadanos no abandonan su apatía o "indiferencia" ante la oportunidad que brindan las elecciones para "expresar" sus preferencias sociales, y entre aquellos que sí votan, los que mantienen su "lealtad" hacia el partido en el poder, al menos últimamente, son cada vez menos en la ZMCM. Pero también hay ciudadanos que han optado por "salirse" del juego electoral antes que responder al llamado a las urnas. A pesar de eso, cada vez un mayor número de ciudadanos reconoce el valor de las elecciones y entiende que es posible "expresar" con el voto su insatisfacción por la situación económica que les afecta, así como llamar la atención sobre el incumplimiento o el viciado desempeño de los gobernantes en turno, como sucedió en 1988 en la región centro de

2 Los bienes públicos "puros" son indivisibles y no excluyentes. Se convierten en impuros cuando se llevan a niveles o escalas locales e interviene la fricción de la distancia en su distribución y consumo (Pinch, 1985). 
México, en 1992 en las elecciones estatales de Chihuahua, y poco antes, en las de Baja California, San Luis Potosí, Guanajuato y Michoacán.

Los anteriores conceptos ${ }^{3}$ que han sido empleados por Hirschman (1970) y algunos de sus seguidores para analizar las reacciones de los consumidores o usuarios respecto de un bien o servicio, o para describir una actitud frente al trabajo, pudieran también servir para ilustrar el comportamiento electoral de los ciudadanos. Para este propósito habría que recurrir al modelo político de Downs (1957), inspirado en el modelo económico de competencia mercantil estable de Hotelling (1929). Según Downs, las elecciones constituyen, para los partidos políticos, un mercado de votos, y para los electores, una oferta de opciones políticas donde canalizar sus preferencias y simpatías. Son relevantes también en este contexto el modelo de Tiebout (1956), que explica las decisiones y el comportamiento espaciales de los sujetos como consecuencia de las diferencias entre unidades político-administrativas en la oferta de bienes y servicios públicos, y la argumentación de Galbraith (1979) acerca de la actitud "resignada" de ciertos grupos o individuos frente a condiciones de pobreza extrema.

Estos modelos permiten analizar el comportamiento de los individuos cuando protestan, cuando se mantienen fieles (social, económica y geográficamente) ante una situación o cuando abandonan sus pautas de consumo, su lugar de trabajo o residencia, y escogen otro producto, marca, club, zona de la ciudad, distrito o circunscripción político-administrativa, etcétera, más acorde con sus gustos o necesidades y con el propósito de elevar sus ingresos reales, aprovechar ventajas económicas, alcanzar estabilidad o libertad políticas y en general mejorar su bienestar y la calidad de su vida y la de sus familias.

La terminología de Hirschman, el modelo de Downs y las ideas que se desprenden de Galbraith y del clásico trabajo de Tiebout, constituyen elementos heurísticos apropiados que nos permiten, desde el punto de vista geográfico, explorar algunos aspectos del comportamiento político del electorado, y las reacciones pre y poselectorales del gobierno y de los partidos políticos.

Sin embargo, cabe distinguir el enfoque geográfico contemporáneo del que resultaría de la geografía tradicional, cuando se trata de estudiar los fenómenos sociales, económicos, culturales y políticos. En ambos casos, desde luego, la relación de tales fenó-

3 Salir (exit), expresar (voice), mostrar lealtad (loyalty) e indiferencia o apatía (neglect) 
menos o procesos con el medio físico (el paisaje y los elementos naturales) es el punto de partida; pero es posible separar analíticamente cada enfoque. 4

La geografía tradicional privilegia la escala y el origen de los fenómenos, de tal suerte que de la interacción de los atributos humanos y geográficos resultan -independientemente de la escala (global, regional, local, etcétera)- espacios o paisajes únicos, distintos de los demás; se trata pues del estudio de las formas particulares en que se manifiestan espacialmente los fenómenos sociales, políticos, económicos y culturales. En cambio, el paradigma geográfico contemporáneo traduce operativamente el concepto de distancia y trata de identificar asociaciones espaciales entre fenómenos. En este sentido, analiza la forma en que un evento u objeto localizado espacialmente permanece, evoluciona y se reproduce en el tiempo, afectando áreas contiguas que se adaptan o se resisten al mismo en función de la presencia o ausencia en ellas de ciertos atributos. De lo anterior se derivan conclusiones positivas (lo que es), o bien predictivas (lo que podría suceder). Las primeras permiten explicar y comparar eventos en espacios y tiempos distintos, y las segundas, tomar decisiones de política. Este enfoque constituye, por así decirlo, la vía de entrada de los geógrafos a la planeación y a la evaluación de políticas.

Con estos elementos conceptuales y metodológicos podemos establecer los alcances de la geografía electoral, aun cuando en este caso sólo en los primeros dos enmarcamos nuestro análisis. De acuerdo a Taylor (1985) y a Bosque Sendra (1988), entre otros, los estudios de geografía electoral se orientan según sus propósitos a:

1) Describir la forma en que se distribuyen geográficamente

${ }^{4}$ La geografía ha insistido tradicionalmente en: $i$ ) conocer todo lo que sucede en la superficie de la tierra, y ii) sostener el principio explicativo de esta fenomenología en la relación hombre-naturaleza. Así, un sistema tradicional de información geográfica estaría formado por el conjunto de reportes (monografías) de los exploradores y expedicionarios al servicio de un Estado y el contenido, finalmente, resultaría en un conocimiento ideográfico basado en el convencimiento de que cada región, área o pedazo de la superficie terrestre es diferente a los demás, en razón del elemento de la ecuación que se privilegie: lo físico o lo cultural. Por el contrario, un enfoque nomotético enfatizaría la generalización empírica y la construcción teórica con fines predictivos. Un sistema de información geográfico asociado a esta perspectiva es el que propone Berry (1964) con la matriz de datos geográficos. Con ella es posible llevar a cabo estudios monográficos, de diferenciación espacial, de asociación y covarianza de variables o lugares, de evolución de lugares o variación de sus atributos en el tiempo, etcétera, desde un enfoque sistémico. 
los resultados electorales, desde el punto de vista estadístico y cartográfico.

2) Explicar los resultados electorales en un área, en función de los atributos que la distinguen; éstos pueden ser geográficos (medio natural ), sociales, económicos, culturales, etcétera.

3) Evaluar los sesgos que una división administrativa determinada o cualquier cambio en ella (organización del territorio en distritos y circunscripciones para fines políticos y electorales), pudiera causar en los resultados finales (Gudgin y Taylor, 1979).

\section{Contexto geográfico megalopolitano}

Hace tiempo, Gottman (1961) describió un fenómeno urbano en la costa noreste de Estados Unidos, que en el caso de la región centro de México "emerge" recientemente: la consolidación de una megalópolis. Ésta resulta del crecimiento demográfico y la expansión física y funcional de la Zona Metropolitana de la Ciudad de México, sobre un extenso territorio. Se organiza así un espacio diferenciado que incluye a numerosas localidades (y otras zonas metropolitanas como las de Toluca y Cuernavaca) y a sus regiones inmediatas, formando una red o sistema complejo de relaciones de dominio-dependencia (funcionales) a partir de mercados regionales y locales más o menos jerarquizados, de factores, insumos, bienes y servicios.

El fenómeno megalopolitano -antes metropolitano- de la ciudad de México puede verse, desde el punto de vista geográfico, a partir de asociaciones espaciales, y a la vez como una diferenciación de áreas o zonas relativamente homogéneas en su interior, que muestran la forma de organización o estructura del espacio urbano y rural de la región. Detrás de ésta subyacen procesos ecológico-demográficos que reflejan el comportamiento social y espacial de la población y pueden comprobarse empíricamente, a través de las mencionadas diferencias entre áreas, atendiendo a los valores de algunas variables socioeconómicas; al cambio en el ritmo de crecimiento demográfico, y a los parámetros que definen diversos gradientes de densidad. Un acercamiento a los datos permite apreciar, en efecto, la sucesión de valores de las tasas de crecimiento poblacional, en forma de olas cuyas crestas van adquiriendo paulatinamente mayores niveles conforme se alejan (en tiempo y distancia) del centro histórico de la ciudad, como un cambio en la función negativa clásica de densidad, cuya pendiente se hace cada vez más horizontal, lo que indica una marcada 
desconcentración y descentralización de población, de funciones especializadas y de actividades económicas urbanas. ${ }^{5}$

No es necesario insistir en que esta dinámica metropolitana y regional afecta los mercados de suelo, vivienda y otros servicios públicos y privados, así como a las características sociodemográficas de la población en general y la fuerza de trabajo en particular. Los tipos de empleo, ocupación y sector de actividad de la población trabajadora, según se localicen en el centro de la ciudad o en la periferia inmediata o más alejada de la misma, van acompañados de transformaciones en la estructura por edad y sexo de la población económicamente activa, lo que, de acuerdo con algunos estudios, modifica sus valores políticos e ideológicos. No debería, por tanto, sorprender que estos cambios se traduzcan en un comportamiento electoral alejado de un modelo ortodoxo de clases sociales, que ahora no refleja las "relaciones sociales" sino más bien las diferencias en la oferta y el consumo de bienes y servicios distribuidos en forma desigual en el ámbito geográfico metropolitano.

De la consideración e interpretación de estos procesos surgen dos propuestas que se debaten con insistencia en la literatura sobre geografía política y electoral. La primera asume que el electorado mantendrá su ideología política de acuerdo con su posición (social y ocupacional) en el trabajo. La segunda, que es más bien el lugar de residencia ${ }^{6}$ el que condiciona, al momento de votar, las aspiraciones sociales y preferencias políticas del elector.

5 Véase Negrete, Graizbord y Ruiz (1993), para una interpretación de estos procesos con valores de algunas variables sociodemográficas censales de 1990 .

6 Entiendo aquí por lugar, no lo que esto significaba para los geógrafos sistemáticos o regionalistas, que siguiendo a Kant entendían el espacio como algo absoluto (i.e. sitio), y por tanto único, sino como un ámbito complejo (social) de relaciones en el tiempo que definen, reproducen y estructuran o, más bien, crean presencia (Giddens, 1981; Gatrell, 1983). Estas relaciones, que ha estudiado el geógrafo desde siempre, no son únicamente entre objetos, o entre hombre y objeto (i.e. hombre-naturaleza), sino entre hombre y hombre (utilizada esta palabra genéricamente). No es posible entrar a esta discusión en el reducido espacio de esta nota o de este artículo, pero quisiera dejar sentada la dificultad implícita de este concepto, señalando la importancia que asignan los geógrafos tanto al significado objetivo como a la percepción, y por tanto al carácter subjetivo de sitio, lugar, milieu, entorno, ámbito, weltschaung, en fin, el fenómeno de la existencia humana y el "cognocimiento" personal de la misma, desde la revolución paradigmática que sacudió a la geografía anglosajona en los años cincuenta. Un intento para llevar estos conceptos al plano operativo, es la clasificación de Sonnenfeld (1972) del "environment" humano en cuatro espacios: el geográfico, que comprende un ámbito externo al hombre independientemente de que lo afecte o no en forma directa; el operativo que corresponde al medio en el que actúa; el perceptivo, que incluye sólo aquellos elementos (objetos, individuos) que es capaz de reconocer y el espacio de su conducta, que involucra la respuesta directa del individuo ante los hechos o eventos que asume como propios. Al respecto considérese la 


\section{Hipótesis de trabajo}

Con los elementos considerados en los párrafos anteriores y sin pretender ir más allá de su carácter heurístico, propongo la tesis de que los ciudadanos o electores metropolitanos tenderán a identificar, en las plataformas que presentan los partidos políticos, las opciones que coincidan con su "estilo de vida", mismo que se asocia regularmente al área o zona de la ciudad en donde se localiza su vivienda. ${ }^{7}$ Este principio permite, en la práctica, analizar resultados electorales asociados a las características socioeconómicas y demográficas de la población residente en una "unidad geográfica de información" que pudiera ser el municipio, un AGEB (área geográfica estadística básica) o el distrito electoral.

Aquí adopto, para el ámbito megalopolitano, una agregación espacial de distritos electorales en zonas geográficas relativamente homogéneas, esto es:

- Ciudad central (CC). Comprende las 4 delegaciones centrales del D.F. que coinciden con 15 distritos electorales.

- Delegaciones contiguas (DC). Se trata de 8 delegaciones subdivididas en 22 distritos electorales.

- Delegaciones sur (DS). Abarca 4 delegaciones que corresponden a 2 distritos electorales.

- Municipios Conurbados (MC). Agrupa 27 municipios en 25 distritos electorales.

- Municipios metropolitanos no conurbados (MNC). Incluye los otros 26 municipios del valle Cuautitlán-Texcoco, agrupados en 2 distritos.

- Zona metropolitana de Toluca (ZMT). Comprende solamente a los municipios de Toluca y Metepec en 2 distritos electorales.

- Resto del Estado de México (REME). Se constituye por el resto de municipios distribuidos en 5 distritos electorales.

- Zona metropolitana de Cuernavaca (ZMC). Corresponde al municipio de Cuernavaca que equivale a un distrito.

- Resto del estado de Morelos (REMO). Abarca al resto de municipios de la entidad, formando los 3 distritos restantes.

"geografía del tiempo" que proponen los geógrafos suecos como Hagerstrand y Carlstein (cit. en Giddens, 1981).

7 El principio de que el comportamiento tanto objetivo como subjetivo de los individuos se asocia con su "ubicación" en el espacio metropolitano, permite dar sentido analítico a los resultados electorales por partido político pues éstos tienen un referente espacial ineludible. 
Asimismo, considero un espectro político integrado por el PAN a la derecha, el PRI al centro, y el FDN (ahora PRD) a la izquierda y establezco las siguientes hipótesis de trabajo para este contexto espacial y político:

H1: En la ZMCM, los votantes de los distritos electorales que corresponden en la actualidad al centro de la ciudad (CC, DC) encontrarán en los partidos políticos tradicionales y conservadores (PRi, PAN) -que mantienen posiciones en favor del mantenimiento del statu quo- sus mejores opciones; en cambio, los residentes en distritos electorales suburbanos (DS), si se trata de áreas consolidadas y con servicios, se inclinarán francamente por la derecha (PAN), y por la izquierda (FDN ahora PRD), constituida por partidos cuyas plataformas son generalmente populistas y muchas veces de contenido utópico, si habitan nuevos asentamientos aún no consolidados y sin servicios (MC o MNC), o bien si son recién llegados o con poco tiempo y conocimiento de la ciudad, bajos niveles educativos y capacidad restringida de participar en los mercados formales de vivienda y de trabajo, etcétera.

H2: En el caso de la región de influencia metropolitana (o resto de la megalópolis) el comportamiento del electorado (que se refleja en el resultado electoral) operará en función del tamaño de las localidades, de las distancias de éstas respecto a la ciudad central o a las capitales estatales, lo que en el caso de nuestro país se refleja en la cantidad y calidad de los medios de transporte intra e interurbanos, de los servicios públicos y, en general, de la infraestructura física y social. En otras palabras, aquellos electores que viven en las ciudades capitales (ZMT o ZMC) o en las más grandes, donde se concentran los servicios públicos locales, votarán por el mantenimiento del statu quo, es decir, a favor de partidos centristas o de derecha, mientras que los habitantes de zonas rurales o marginales (REME, REMO o MNC) harán lo contrario. 8

${ }^{8}$ En México, la población rural ha sido desde siempre manipulada política y electoralmente por el partido en el poder, pero existen movimientos en el campo que se han alejado ("salido") de su tutela y control. Algunos de ellos empiezan a expresar su desencanto votando por las opciones que ofrecen otros partidos. En el caso de la población de las zonas marginadas, ésta no ha sido capaz de organizarse políticamente o bien se caracteriza por su tendencia al clientelismo, aunque es necesario reconocer que hay múltiples ejemplos de cohesión y articulación política de agrupaciones civiles y sindicales que aún no han podido, por su carácter muchas veces efímero o por su incipiente tradición y cultura políticas, constituir, aglutinar o controlar organizaciones políticas formales, es decir, partidos. 
La velocidad del crecimiento demográfico en la ZMCM y la expansión continua de su área de influencia cotidiana (que involucra el lugar de trabajo con el de residencia) hacen difícil la comprobación rigurosa o el rechazo categórico de las hipótesis anteriores. Sin embargo, en los resultados gráficos que se presentan y con la debida cautela, dados el carácter exploratorio de este trabajo, el corte sincrónico del análisis y el nivel de agregación aquí adoptado, puede reconocerse que lo que pasa en otras zonas metropolitanas (o megalópolis) del mundo también sucede en ésta.

\section{Resultados empíricos}

Como se aprecia en las gráficas 1 y 2 , y el cuadro 1 que acompañan al texto, las tendencias del voto en 1988 en favor de la izquierda (FDN) y de la derecha (PAN) son, en términos espaciales, diametralmente opuestas.

GRÁFICA 1

Región central. Tendencia espacial del voto, 1988

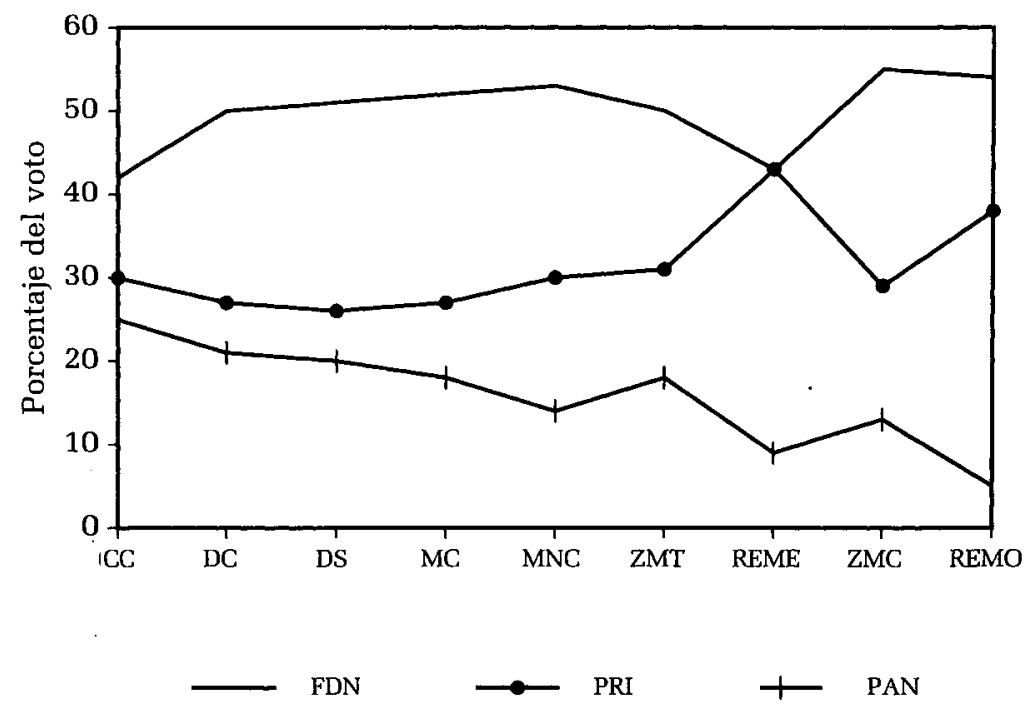


GRÁFICA 2

Región central. Distribución del voto según zona, 1988

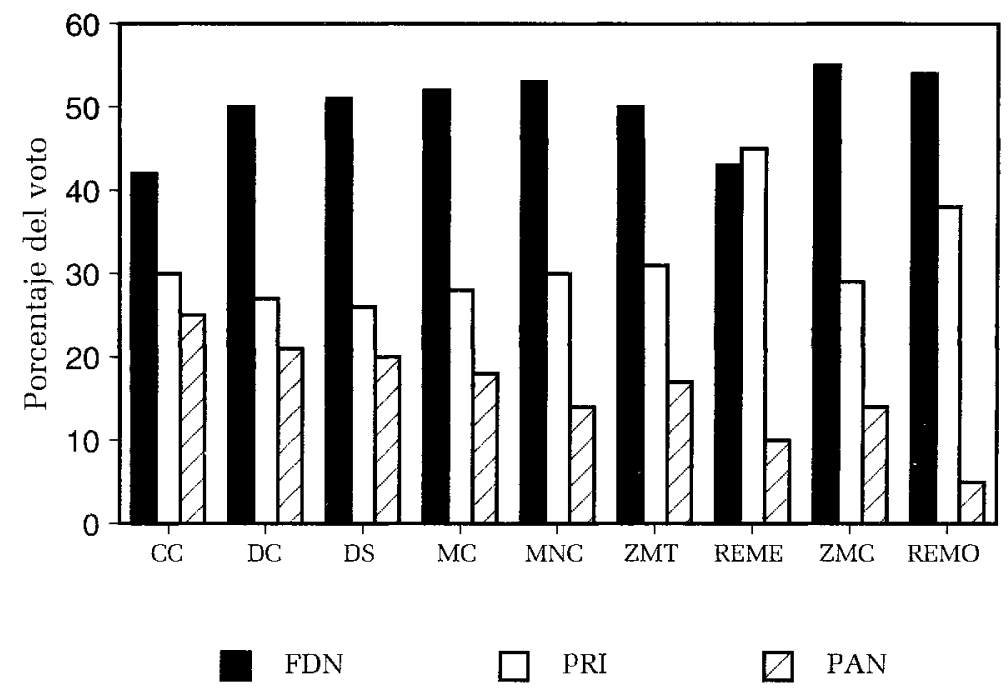

El voto para el pAN muestra una caída conforme nos alejamos de la ciudad central (CC), con claros incrementos en las dos capitales estatales (ZMT y ZMC). Lo anterior comprueba el carácter urbano de sus simpatizantes. ${ }^{9}$

En cambio, para el FDN el menor valor se presenta en el centro de la ciudad, incrementándose el porcentaje de votos a su favor conforme nos alejamos hacia la periferia inmediata de los municipios conurbados y no conurbados ( $M C$ y MNC), donde vive la población de bajos ingresos, recién llegada a la ciudad; en viviendas precarias y sin acceso a servicios e infraestructura. Se aprecia también una caída del valor porcentual del voto a su favor en la

9 Digo simpatizantes, pues a pesar de que este partido logra en todas las elecciones una nutrida votación, que lo ha convertido en la segunda fuerza electoral del país, sus propios dirigentes se quejan de la bajísima afiliación formal con que cuenta. 


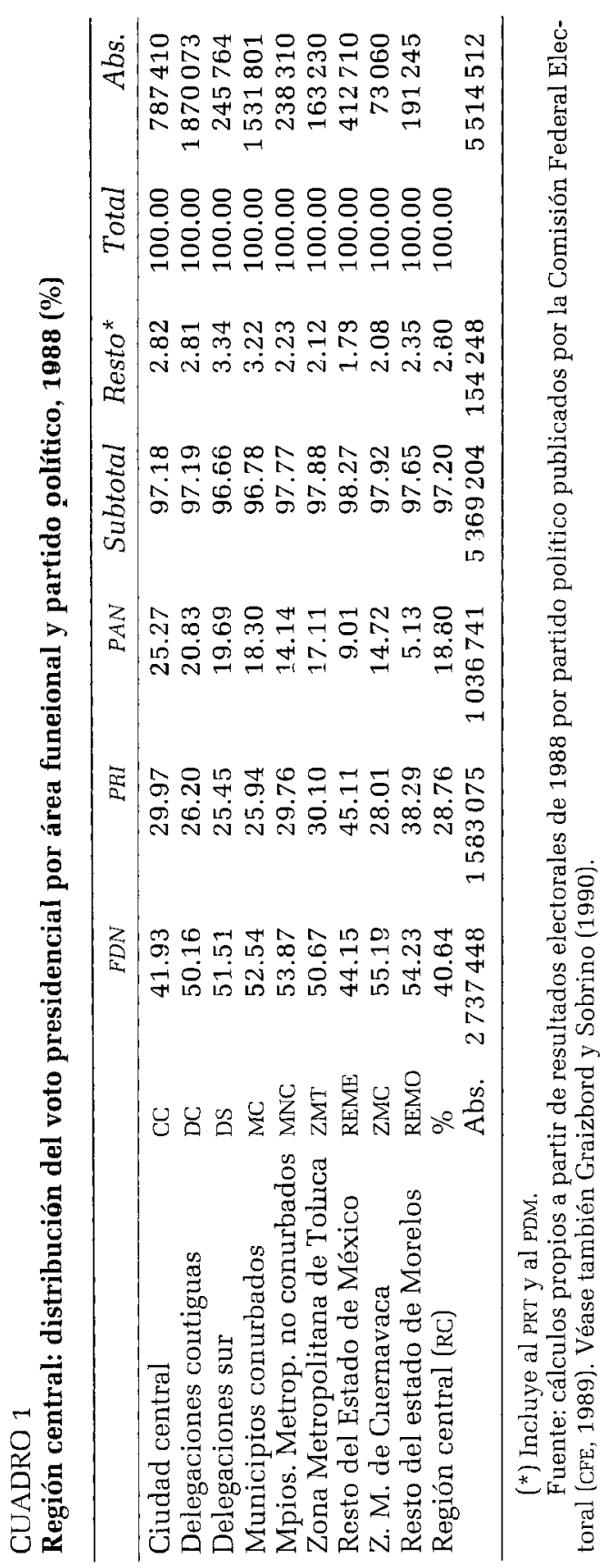


Zona Metropolitana de Toluca (ZMT), que se inclina a la derecha. Asimismo, se votó por ese partido en el resto del Estado de México (REME), donde la población rural perteneciente a diversos grupos étnicos se caracteriza por su incipiente cultura política y sus bajos niveles educativos; dedicada mayormente a las actividades agrícolas, habiendo soportado históricamente un dominio absoluto del PRI y un control social, político y económico de sus representantes (caciques). El gradiente vuelve a subir y muestra el mayor valor porcentual en la Zona Metropolitana de Cuernavaca (ZMC) y en el ámbito rural de Morelos (REMO), lo que indica, nuevamente, una abrumadora simpatía del electorado de esa entidad por la izquierda y lo que ésta representa, o bien expresa la convicción de que ha sido "traicionado" por el régimen, aunque también refleja la avanzada organización política del campesinado morelense, protagónico en su momento de la Revolución mexicana.

Si ahora observamos el gradiente que representa el voto al PRI, podemos percatarnos de que, tanto el electorado de la ciudad central (CC) como el de los municipios metropolitanos no conurbados (MNC), son los que le mostraron su "lealtad" y simpatía. El punto sobresaliente en el resto del Estado de México (REME), donde el PRI obtuvo una gran cantidad de votos, incluso en el marco de una abrumadora derrota, indica la capacidad de movilizar al campesinado que aún agrupa la CNC (sector campesino del partido oficial) en esa región. Puede verse que esto es válido también, aunque no con la misma intensidad, para el resto de Morelos (REMO). En Cuernavaca, la capital de esa entidad, el voto en favor del PRI cayó proporcionalmente a niveles comparables con los más bajos que obtuvo este partido en la ZMCM, que corresponden a las delegaciones centrales y sur del D.F. (DC y DS) y a los municipios conurbados (MC) del Estado de México.

\section{Reflexiones finales}

Una descripción somera, como la que aquí presento, de la distribución del voto presidencial de 1988 en la megalópolis, no es quizá suficiente. Sin embargo, su nitidez sorprende y permite aceptar, en principio, las hipótesis formuladas, aunque la segunda sólo parcialmente.

Cierto resulta, que en la actualidad es difícil sostener el supuesto sociológico de que la edad, el sexo y la ubicación espacial 
se desvanecen, por así decirlo, en la categoría "clase social" y que sólo este atributo explicaría variaciones sistemáticas en el comportamiento sociopolítico de los individuos (como consumidores, jefes de familia, electores, etcétera).

La premisa "dime por quién votas y te diré a que clase social perteneces" es cada vez menos válida, al menos para el ámbito urbano y metropolitano. Ni siquiera la educación, dada su difusión masiva, permite una certera predicción de la conducta del individuo. "Dime donde vives y te diré como votas" sería, en este contexto, el punto de partida para predecir en el futuro los resultados electorales en las numerosas y cada vez más grandes y complejas zonas metropolitanas del país.

Como señalaba Bell (1977), los aspectos más idiosincráticos de la experiencia personal y el "curso de vida" individual adquieren más importancia que los atributos sociales en la confección del estilo de vida de una persona; de tal suerte que con la disolución de la estructura tradicional de clases, los individuos son y desean ser identificados no por su relación de trabajo o su ocupación, sino por sus gustos y estilos de vida. Así, más que la ocupación -o pertenencia a una clase social en términos marxistas-, es la ubicación del individuo en el espacio metropolitano (megalopolitano), aquí representado analíticamente por las distintas áreas funcionales en que se dividió a la RC, lo que refleja y condiciona su conducta y preferencias sociales, en este caso expresadas por el voto a un partido político, y por ende, en los resultados electorales.

La geografía del voto, como resultado del comportamiento de los electores, exhibe este fenómeno reciente de la conducta política de los ciudadanos en los conglomerados urbanos, independientemente de los niveles de desarrollo o del grado de democracia de los sistemas, como las experiencias políticas y electorales recientes en la Europa oriental parecen confirmar. En efecto, la Zona Metropolitana de la Ciudad de México - la megalópolis del centro del país- no representa, desde el punto de vista del comportamiento de la población en general o del electorado, en particular, un caso único. Habrá que esperar otras elecciones federales, en el marco de una legítima reforma electoral, para elevar esta conclusión a un estatus más general con valor predictivo, y por tanto, de interés para los partidos políticos, el gobierno, los ciudadanos megalopolitanos y los estudiosos de estos fenómenos. 
Bibliografía

Bell, D. (1976), The Coming of Post-Industrial Society, Basic Books.

Berry, B. (1964), "Approaches to Regional Analysis: A Synthesis", en Annals, Association of American Geographers, vol. 54, pp. 2-11.

Bosque Sendra, J. (1988), Geografía electoral, Síntesis.

Downs, A. (1957), An Economic Theory of Democracy, Nueva York, Harper and Brothers.

Galbraith, J. K. (1979), The Nature of Mass Poverty, Harvard University Press.

Garza, G. (1987), "El futuro de la ciudad de México. Megalópolis emergente", en G. Garza (comp.) Atlas de la ciudad de México, México, El Colegio de México/Departamento del Distrito Federal.

Gatrell, A. (1983), Distance and Space: A Geographical Perspective, Clarendon.

Giddens, A. (1981), A Contemporary Critique of Historical Materialism, University of California Press.

Gottman, J. (1961), Megalopolis, Twentieth Century Fund.

Graizbord, B. y L. J. Sobrino (1990), "Geografía política de la región metropolitana de la ciudad de México", en Actas Latinoamericanas de Varsovia, 11, pp. 227-251.

Gudgin G. y P. Taylor (1979), Seats, Votes and the Spatial Organization of Elections, Pion.

Harvey, D. (1973), Explanation in Geography, Edward Arnold.

Hirschman, A.O. (1970), Exit, Voice and Loyalty, Harvard University Press.

- (1981), "Exit, Voice and the State", en A.O. Hirschman (ed.,), Essays in Trespassing, Cambridge University Press.

Holt-Jensen, A. (1980), Geography, its History and Concepts, Nueva York, Harper and Row.

Hotelling, H. (1929), "Stability in Competition", en Economic Journal, vol. 39, pp. 41-57.

Negrete, M. E., B. Graizbord y C. Ruiz (1993), "Población, espacio y medio ambiente en la Zona Metropolitana de la Ciudad de México" Cuadernos de Trabajo, núm. 2, México, LEAD-México-CEDDU-El Colegio de México.

Pinch, S. (1985), Cities and Services, Routledge and Kegan Paul.

Sonnenfeld, J. (1972), "Geography, Perception, and the Behavioral Environment", en P. English and R. Mayfield (eds.), Man, Space and the Environment, Oxford University Press.

Taylor, P. (1985), "The Geography of Elections", en M. Pacione (ed.), Progress in Political Geography, Croom Helm.

Thurow, L. (1971), "The Income Distribution as a pure Public Good", en Quarterly Journal of Economics, vol. 85, pp. 327-336.

Tiebout. C. (1956), "A Pure Theory of Local Expenditures", en Journal of Political Economy, vol. 64, pp. 416-424. 